\title{
Research Article \\ Optimal Control for a Class of Chaotic Systems
}

\author{
Jianxiong Zhang and Wansheng Tang \\ Institute of Systems Engineering, Tianjin University, Tianjin 300072, China \\ Correspondence should be addressed to Jianxiong Zhang, jxzhang@tju.edu.cn
}

Received 19 October 2011; Revised 17 January 2012; Accepted 10 February 2012

Academic Editor: Chuanhou Gao

Copyright (C) 2012 J. Zhang and W. Tang. This is an open access article distributed under the Creative Commons Attribution License, which permits unrestricted use, distribution, and reproduction in any medium, provided the original work is properly cited.

This paper proposes the optimal control methods for a class of chaotic systems via state feedback. By converting the chaotic systems to the form of uncertain piecewise linear systems, we can obtain the optimal controller minimizing the upper bound on cost function by virtue of the robust optimal control method of piecewise linear systems, which is cast as an optimization problem under constraints of bilinear matrix inequalities (BMIs). In addition, the lower bound on cost function can be achieved by solving a semidefinite programming (SDP). Finally, numerical examples are given to illustrate the results.

\section{Introduction}

As a very interesting nonlinear phenomenon, chaos has been widely applied in many areas, such as secure communication, signal generator design, biology, economics, and many other engineering systems, which has been researched thoroughly over the past two decades [1]. Recently, chaos control of chaotic systems has become an active research topic [2]. In general, there are several schemes to achieve the control of continuous time chaotic systems, such as OGY method [3], parametric resonance method [4], adaptive feedback method $[5,6]$, delay feedback method [7], backstepping design method [8], fractional controller design method [9], sliding mode control method [10, 11], internal model approach [12], impulsive control approach [13], as well as linear and nonlinear feedback control methods [14-17]. However, most of the existing methods were used to achieve chaos control either by employing the linearization scheme in the neighborhood of the objective point which is difficult to accomplish the global analysis, or by applying the nonlinear feedback controller which often limits practical applications. Based on the fuzzy control theory, Tanaka et al. [18] studied the feedback control of chaotic systems. The result formulated in terms of linear matrix inequalities (LMIs, [19]) was convenient to solve, but the controller design for the associated fuzzy systems was fulfilled by virtue of global quadratic Lyapunov function which is conservative in the control synthesis. 
As pointed out in [20], piecewise linear systems, which can approximate general nonlinear systems to any degree of accuracy, can be analyzed based on piecewise quadratic Lyapunov function technique that introduces more flexibility than the classical global quadratic Lyapunov function technique. Thus, the piecewise linear systems provide a powerful way of analysis and synthesis for nonlinear systems. Chaotic systems belong to complex nonlinear systems. In fact, it is significant to design a practicable piecewise linear feedback controller to stabilize globally a chaotic system with a performance measure for the control synthesis. We recently [21] proposed a new chaotic system and designed a piecewise linear feedback controller to stabilize globally the new system based on piecewise linear systems method. So far, there have been very few results dealing with the optimal control for chaotic systems. In this paper, we investigate the problem of designing piecewise linear feedback controller to stabilize a class of chaotic systems, and meanwhile minimize a quadratic cost function for the closed-loop systems. Particularly, in this paper, a class of chaotic systems are converted to uncertain piecewise linear systems. Then, based on piecewise quadratic Lyapunov function technique and Hamilton-Jacobi-Bellman (HJB) inequality method, the optimal chaos control via piecewise linear state feedback controller is studied. It is shown that the optimal controller minimizing the upper bound on cost function can be obtained by solving an optimization problem under constraints of bilinear matrix inequalities (BMIs). The lower bound on cost function can be attained by solving a semidefinite programming (SDP). If the upper and lower bounds obtained are sufficiently tight, it is concluded that the associated solutions achieve or get close to optimality.

This paper is organized as follows. In Section 2, the optimal control problem of chaotic systems is introduced. In Section 3, the optimal control for a class of chaotic systems via piecewise linear state feedback controller is proposed. The upper bound and lower bound on cost function are designed. Illustrative examples are given in Section 4, and the conclusion is drawn in Section 5.

Throughout this paper, a real symmetric matrix $P>0(\geq 0, \leq 0)$ denotes $P$ being a positive definite (positive semidefinite, or negative semidefinite) matrix, and $A>B$ means $A-B>0$. I denotes an identity matrix of appropriate dimension. The superscript " $T$ " represents the transpose of a matrix. Matrices, if their dimensions are not explicitly stated, are assumed to have compatible dimensions for algebraic operations.

\section{Problem Formulation}

Consider the chaotic system of the form:

$$
\dot{\mathbf{x}}=A \mathbf{x}+F(\mathbf{x})+B \mathbf{u},
$$

where $A$ and $B$ are constant matrices, $\mathbf{x} \in \mathfrak{R}^{n}$ is the state vector, $\mathbf{u} \in \mathfrak{R}^{m}(m \leq n)$ is the control input variable, and the nonlinear term $F(\mathbf{x}) \in \mathfrak{R}^{n}$ is assumed to satisfy Lipschitz continuity condition, uniform or local, and $F(0)=0$.

Associated with this system is the cost function:

$$
J=\int_{0}^{\infty}\left(\mathbf{x}^{T}(t) Q \mathbf{x}(t)+\mathbf{u}^{T}(t) R \mathbf{u}(t)\right) \mathrm{d} t
$$

where $Q>0, R>0$ are given weighting matrices. 
The goal of this paper is to design a state feedback law $\mathbf{u}(t)$ stabilizing the chaotic system (2.1) and meanwhile minimizing the cost function (2.2).

It is known that the control law $\mathbf{u}(t)$ can be derived from the solution to the associated HJB equation. However, generally speaking, the HJB equation corresponding to a general nonlinear system is notoriously hard to solve. Many numerical methods have been devised for the solution of optimal control problems but tended to suffer from combinatorial explosion. Piecewise linear systems, which can approximate nonlinear systems to any degree of accuracy, provide a powerful means of analysis for nonlinear systems. By virtue of HJB inequalities rather than equations, the authors in $[20,22]$ have investigated the state feedback optimal control of piecewise linear systems. It was shown that the upper bound on piecewise quadratic cost function can be obtained by solving a nonconvex BMIs problem, and the lower bound on cost function can be obtained by solving an SDP. Motivated by this, we first convert the chaotic system (2.1) to the form of uncertain piecewise linear systems and then extend the corresponding results of optimal control for the ordinary piecewise linear systems in [20] to the case of uncertain piecewise linear systems. Thus, we can achieve the optimal control for the original chaotic system.

Note that the nonlinear term $F(\mathbf{x})$ in system (2.1) can be approximated by a piecewise linear function as follows:

$$
F(\mathbf{x})=K_{i} \mathbf{x}+a_{i}+\Delta_{i}(\mathbf{x}), \quad \mathbf{x} \in X_{i}, i \in \mathbb{I},
$$

where $K_{i} \in \mathfrak{R}^{n \times n}, a_{i} \in \mathfrak{R}^{n}$ are some given parameters, $\left\{X_{i}\right\}_{i \in \mathbb{I}} \subseteq \mathfrak{R}^{n}$ denotes a partition of the state space of chaotic system, $\mathbb{I}$ is the index set, and $\Delta_{i}(\mathbf{x})$ is the approximation error, which can be regarded as uncertainties in the system. Then, it is obvious that system (2.1) can be converted to the uncertain piecewise linear system:

$$
\dot{\mathbf{x}}=\left(A+K_{i}\right) \mathbf{x}+a_{i}+\Delta_{i}(\mathbf{x})+B \mathbf{u}, \quad \mathbf{x} \in X_{i}, i \in \mathbb{I} .
$$

It is worth mentioning that system (2.1) can represent a large class of chaotic systems such as Genesio-Tesi chaotic system [23], Coullet chaotic system [24], Chua's Circuit system [25], and the new chaotic systems presented in [21,26]. A simple but typical case is the threedimensional chaotic system with the nonlinear term $F(\mathbf{x})$ taking the following form:

$$
F(\mathbf{x})=\left[0,0, f\left(x_{1}\right)\right]^{T},
$$

where $f\left(x_{1}\right)$ is the nonlinear term in the 3rd dimension of the system and can be approximated by a piecewise linear function as

$$
f\left(x_{1}\right)=k_{i} x_{1}+l_{i}+\delta_{i}\left(x_{1}\right), \quad \mathbf{x} \in X_{i}, \quad i \in \mathbb{I},
$$

where $k_{i}, l_{i} \in \mathfrak{R}$ are some given parameters, $\delta_{i}\left(x_{1}\right)$ is the approximation error. Then, system (2.1) with the nonlinear term (2.5) can be converted to the form of the uncertain piecewise linear system (2.4) as

$$
\dot{\mathbf{x}}=A_{i} \mathbf{x}+a_{i}+\Delta_{i}+B \mathbf{u}, \quad \mathbf{x} \in X_{i}, i \in \mathbb{I}
$$


with

$$
A_{i}=A+\left[\begin{array}{lll}
0 & 0 & 0 \\
0 & 0 & 0 \\
k_{i} & 0 & 0
\end{array}\right], \quad a_{i}=\left[\begin{array}{l}
0 \\
0 \\
l_{i}
\end{array}\right], \quad \Delta_{i}=\left[\begin{array}{c}
0 \\
0 \\
\delta_{i}\left(x_{1}\right)
\end{array}\right]
$$

\section{State Feedback Optimal Control of Systems}

Without loss of generality, consider the uncertain piecewise linear system of the form

$$
\dot{\mathbf{x}}(t)=\left(A_{i}+\Delta A_{i}\right) \mathbf{x}(t)+\left(B_{i}+\Delta B_{i}\right) \mathbf{u}(t)+a_{i}+\Delta a_{i}
$$

for $\mathbf{x}(t) \in X_{i}$, where $\left\{X_{i}\right\}_{i \in \mathbb{I}} \subseteq \mathfrak{R}^{n}$ denotes a partition of the state space into a number of polyhedral cells, $\mathbb{I}$ is the index set of the cells, $\left(A_{i}, B_{i}, a_{i}\right)$ is the $i$ th nominal local model of the system, $a_{i}$ is the offset term. $\Delta A_{i}, \Delta B_{i}$, and $\Delta a_{i}$ represent parametric perturbations in the system state matrix, input matrix, and offset term of the $i$ th nominal local model, respectively, and are assumed to be of the following form:

$$
\left[\Delta A_{i}, \Delta B_{i}, \Delta a_{i}\right]=M_{i} H\left[N_{A_{i}}, N_{B_{i}}, N_{a_{i}}\right]
$$

where $H \in \mathfrak{R}^{i \times j}$ is an uncertain matrix bounded by $H^{T} H \leq I$, and $M_{i}, N_{A_{i}}, N_{B_{i}}, N_{a_{i}}$ are known constant matrices of appropriate dimensions which specify how the elements of the nominal matrices $A_{i}, B_{i}$, and $a_{i}$ are affected by the uncertain parameters in $H$.

Define $\mathbb{I}_{0} \subseteq \mathbb{I}$ as the set of indices for cells that contain origin and $\mathbb{I}_{1} \subseteq \mathbb{I}$ the set of indices for cells that do not contain the origin. It is assumed that $a_{i}=\Delta a_{i}=0$ for all $i \in \mathbb{I}_{0}$.

For any given initial condition $\mathbf{x}(0)=\mathbf{x}_{0}$, and input signals $\mathbf{u}$, it is assumed that system (3.1) has a unique solution, and there is no sliding mode. Note that with possible discontinuities in $A_{i} \mathbf{x}$ across the boundaries of the partitions, the solution of system (3.1) may be just continuous and piecewise $C^{1}$. For a definition of the state trajectory of the system in (3.1) refer to [20] for details.

For convenience, the following notations are introduced:

$$
\begin{gathered}
\overline{\mathbf{x}}=\left[\begin{array}{l}
\mathbf{x} \\
1
\end{array}\right], \quad \bar{A}_{i}=\left[\begin{array}{cc}
A_{i} & a_{i} \\
0 & 0
\end{array}\right], \quad \bar{B}_{i}=\left[\begin{array}{c}
B_{i} \\
0
\end{array}\right], \quad \bar{M}_{i}=\left[\begin{array}{c}
M_{i} \\
0
\end{array}\right], \quad \bar{N}_{A_{i}}=\left[\begin{array}{ll}
N_{A_{i}}, & N_{a_{i}}
\end{array}\right], \\
\Delta \bar{A}_{i}=\left[\begin{array}{cc}
\Delta A_{i} & \Delta a_{i} \\
0 & 0
\end{array}\right]=\bar{M}_{i} H \bar{N}_{A_{i}}, \quad \Delta \bar{B}_{i}=\left[\begin{array}{c}
\Delta B_{i} \\
0
\end{array}\right]=\bar{M}_{i} H N_{B_{i}},
\end{gathered}
$$

then system (3.1) can be expressed as

$$
\dot{\overline{\mathbf{x}}}(t)=\left(\bar{A}_{i}+\Delta \bar{A}_{i}\right) \overline{\mathbf{x}}(t)+\left(\bar{B}_{i}+\Delta \bar{B}_{i}\right) \mathbf{u}(t), \quad i \in \mathbb{I} .
$$


Associated with this system is the following cost function:

$$
J=\int_{0}^{\infty}\left(\mathbf{x}^{T}(t) Q_{i} \mathbf{x}(t)+\mathbf{u}^{T}(t) R_{i} \mathbf{u}(t)\right) \mathrm{d} t
$$

where $i$ is defined so that $\mathbf{x}(t) \in X_{i}$, and $Q_{i}>0, R_{i}>0$ are given weighting matrices.

Note that if $Q_{i}, R_{i}$ in (3.5) are set to be the same, respectively, for every $i \in \mathbb{I}$, the cost function (3.5) will reduce to (2.2). In addition, the matrix $\bar{Q}_{i}=\operatorname{diag}\left\{Q_{i}, 0\right\} \in \mathfrak{R}^{(n+1) \times(n+1)}$ is introduced, which will be used in the sequel.

As noted in [20], to find a piecewise Lyapunov function that is continuous across region boundaries, the matrices $\bar{F}_{i}=\left[F_{i}, f_{i}\right], i \in \mathbb{I}$ with $f_{i}=0$ for $i \in \mathbb{I}_{0}$ should be constructed, which are used to characterize the boundaries between the regions:

$$
\bar{F}_{i} \overline{\mathbf{x}}=\bar{F}_{j} \overline{\mathbf{x}}, \quad \mathbf{x} \in X_{i} \cap X_{j}, i, j \in \mathbb{I} .
$$

Then, the piecewise Lyapunov function candidates that are continuous across the region boundaries can be parameterized as

$$
V(\mathbf{x})= \begin{cases}\mathbf{x}^{T} P_{i} \mathbf{x}, & \mathbf{x} \in X_{i}, i \in \mathbb{I}_{0}, \\ \overline{\mathbf{x}}^{T} \bar{P}_{i} \overline{\mathbf{x}}, & \mathbf{x} \in X_{i}, i \in \mathbb{I}_{1},\end{cases}
$$

with $P_{i}=F_{i}^{T} S F_{i}$ and $\bar{P}_{i}=\bar{F}_{i}^{T} S \bar{F}_{i}$, where $S$ is a symmetric matrix which characterizes the free parameters of the Lyapunov function candidates.

Note the form of $\bar{P}_{i}$ and the characteristics of the matrices $\bar{F}_{i}$. The continuity of the Lyapunov function $V(\mathbf{x})$ across the partition boundaries is ensured from (3.6) and (3.7).

The $S$-procedure has been used in $[20,22]$ to reduce the conservatism of the stability result. Specifically, the matrices $\bar{E}_{i}=\left[E_{i}, e_{i}\right], i \in \mathbb{I}$ with $e_{i}=0$ for $i \in \mathbb{I}_{0}$, such that

$$
\bar{E}_{i} \overline{\mathbf{x}} \geq 0, \quad \mathbf{x} \in X_{i}, \quad i \in \mathbb{I},
$$

should be constructed to verify the positivity of a piecewise quadratic function of the form (3.7) on a polyhedral partition. It should be noted that the above vector inequalities imply that each entry of the vector is nonnegative.

A systematic procedure for constructing the matrices $\bar{E}_{i}, \bar{F}_{i}$ for a given piecewise linear system was suggested in [20].

Consider the following piecewise linear feedback control law:

$$
\mathbf{u}=-L_{i} \mathbf{x}-l_{i}:=-\bar{L}_{i} \overline{\mathbf{x}}, \quad \mathbf{x} \in X_{i}, i \in \mathbb{I},
$$

with $l_{i}=0$ for $i \in \mathbb{I}_{0}$.

In general, the control law of form (3.9) will bring more flexibility in stability analysis than that of the ordinary linear feedback form. However, this control law may be discontinuous and give rise to sliding modes [20]. To avoid this case, we should construct 
the control law continuously across subspace boundaries and take the feedback gain matrix $\bar{L}_{i}$ as follows

$$
\bar{L}_{i}=L^{T} \bar{F}_{i}, \quad i \in \mathbb{I},
$$

where $L$ is a parameter matrix characterizing the free parameters of the state feedback controller, and $\bar{F}_{i}$ is the matrix defined in (3.6). It should be pointed out that the gain matrix $\bar{L}_{i}$ should take the form of $L_{i}=L^{T} F_{i}$ for $i \in \mathbb{I}_{0}$.

Substituting the control law (3.9) into system (3.4), we can get the following closedloop system:

$$
\begin{gathered}
\dot{\mathbf{x}}(t)=\left(A_{i}+\Delta A_{i}-\left(B_{i}+\Delta B_{i}\right) L_{i}\right) \mathbf{x}(t), \quad \text { for } i \in \mathbb{I}_{0}, \\
\dot{\overline{\mathbf{x}}}(t)=\left(\bar{A}_{i}+\Delta \bar{A}_{i}-\left(\bar{B}_{i}+\Delta \bar{B}_{i}\right) \bar{L}_{i}\right) \overline{\mathbf{x}}(t), \quad \text { for } i \in \mathbb{I}_{1} .
\end{gathered}
$$

Our goal in this section is to find a parameter matrix $L$ to stabilize system (3.11) and meanwhile minimize the cost function (3.5). Before presenting the main results of this paper, we introduce the following lemmas.

Lemma 3.1 (Johansson and Rantzer [22]). Consider symmetric matrices $S, U_{i}$, and $W_{i}$ such that $U_{i}$ and $W_{i}$ have nonnegative entries, while $P_{i}=F_{i}^{T} S F_{i}, i \in \mathbb{I}_{0}$ and $\bar{P}_{i}=\bar{F}_{i}^{T} S \bar{F}_{i}, i \in \mathbb{I}_{1}$, satisfy

$$
\begin{gathered}
A_{i}^{T} P_{i}+P_{i} A_{i}+E_{i}^{T} U_{i} E_{i}<0, \\
E_{i}^{T} W_{i} E_{i}<P_{i}
\end{gathered}
$$

for $i \in \mathbb{I}_{0}$, and

$$
\begin{gathered}
\bar{A}_{i}^{T} \bar{P}_{i}+\bar{P}_{i} \bar{A}_{i}+\bar{E}_{i}^{T} U_{i} \bar{E}_{i}<0, \\
\bar{E}_{i}^{T} W_{i} \bar{E}_{i}<\bar{P}_{i},
\end{gathered}
$$

for $i \in \mathbb{I}_{1}$, then every continuous and piecewise $C^{1}$ trajectory $\mathbf{x}(t)$ of system (3.4) with $\Delta A_{i}=0$, $\Delta a_{i}=0$ and $u=0$ for all $t>0$ tends to zero exponentially.

Lemma 3.2 (Xie [27]). Given matrices $G, M$, and $N$ of appropriate dimensions with $G$ symmetric, then $G+M H N+N^{T} H^{T} M^{T}<0$ for all matrices $H$ satisfying $H^{T} H \leq I$, if and only if there exists some $\varepsilon>0$ such that

$$
G+\varepsilon^{-1} M M^{T}+\varepsilon N^{T} N<0 .
$$

Motivated by the result in [20], we can get the upper bound on the cost function (3.5) for uncertain piecewise linear systems based on the HJB inequality method. The result is presented as follows. 
Theorem 3.3. Consider the closed-loop uncertain system (3.11) with $\mathbf{x}_{0} \in X_{i_{0}}$. If there exist a set of constants $\varepsilon_{i}>0$ and symmetric matrices $S, U_{i}$, and $W_{i}$ such that $U_{i}$ and $W_{i}$ have nonnegative entries, while $P_{i}=F_{i}^{T} S F_{i}, i \in \mathbb{I}_{0}$, and $\bar{P}_{i}=\bar{F}_{i}^{T} S \bar{F}_{i}, i \in \mathbb{I}_{1}$, satisfy

$$
\left[\begin{array}{cccc}
\Phi_{i} & \varepsilon_{i}\left(N_{A_{i}}-N_{B_{i}} L_{i}\right)^{T} & P_{i} M_{i} & L_{i}^{T} \\
\varepsilon_{i}\left(N_{A_{i}}-N_{B_{i}} L_{i}\right) & -\varepsilon_{i} I & 0 & 0 \\
M_{i}^{T} P_{i} & 0 & -\varepsilon_{i} I & 0 \\
L_{i} & 0 & 0 & -R_{i}^{-1}
\end{array}\right]<0
$$

for $i \in \mathbb{I}_{0}$,

$$
\left[\begin{array}{cccc}
\bar{\Phi}_{i} & \varepsilon_{i}\left(\bar{N}_{A_{i}}-N_{B_{i}} \bar{L}_{i}\right)^{T} & \bar{P}_{i} \bar{M}_{i} & \bar{L}_{i}^{T} \\
\varepsilon_{i}\left(\bar{N}_{A_{i}}-N_{B_{i}} \bar{L}_{i}\right) & -\varepsilon_{i} I & 0 & 0 \\
\bar{M}_{i}^{T} \bar{P}_{i} & 0 & -\varepsilon_{i} I & 0 \\
\bar{L}_{i} & 0 & 0 & -R_{i}^{-1}
\end{array}\right]<0
$$

for $i \in \mathbb{I}_{1}$, where

$$
\begin{gathered}
\Phi_{i}:=\left(A_{i}-B_{i} L_{i}\right)^{T} P_{i}+P_{i}\left(A_{i}-B_{i} L_{i}\right)+E_{i}^{T} U_{i} E_{i}+Q_{i}, \\
\bar{\Phi}_{i}:=\left(\bar{A}_{i}-\bar{B}_{i} \bar{L}_{i}\right)^{T} \bar{P}_{i}+\bar{P}_{i}\left(\bar{A}_{i}-\bar{B}_{i} \bar{L}_{i}\right)+\bar{E}_{i}^{T} U_{i} \bar{E}_{i}+\bar{Q}_{i},
\end{gathered}
$$

then the closed-loop system is globally exponentially stable, and the cost function (3.5) satisfies

$$
J \leq \inf _{S, U_{i}, W_{i}, \varepsilon_{i}} \overline{\mathbf{x}}_{0}^{T} \bar{P}_{i_{0}} \overline{\mathbf{x}}_{0}
$$

Proof. By Schur complement [19], the first inequality of (3.15) is equivalent to

$$
\Phi_{i}+L_{i}^{T} R_{i} L_{i}+\varepsilon_{i}^{-1} P_{i} M_{i} M_{i}^{T} P_{i}+\varepsilon_{i}\left(N_{A_{i}}-N_{B_{i}} L_{i}\right)^{T}\left(N_{A_{i}}-N_{B_{i}} L_{i}\right)<0 .
$$

Note the definitions of (3.3) and (3.17). By virtue of Lemma 3.2, inequality (3.19) is equivalent to

$$
\left(A_{i}+\Delta A_{i}-\left(B_{i}+\Delta B_{i}\right) L_{i}\right)^{T} P_{i}+P_{i}\left(A_{i}+\Delta A_{i}-\left(B_{i}+\Delta B_{i}\right) L_{i}\right)+E_{i}^{T} U_{i} E_{i}+Q_{i}+L_{i}^{T} R_{i} L_{i}<0
$$


Along a similar proof technique as used above, it can also be shown that the first inequality of (3.16) is equivalent to

$$
\left(\bar{A}_{i}+\Delta \bar{A}_{i}-\left(\bar{B}_{i}+\Delta \bar{B}_{i}\right) \bar{L}_{i}\right)^{T} \bar{P}_{i}+\bar{P}_{i}\left(\bar{A}_{i}+\Delta \bar{A}_{i}-\left(\bar{B}_{i}+\Delta \bar{B}_{i}\right) \bar{L}_{i}\right)+\bar{E}_{i}^{T} U_{i} \bar{E}_{i}+\bar{Q}_{i}+\bar{L}_{i}^{T} R_{i} \bar{L}_{i}<0
$$

where $\bar{Q}_{i}=\operatorname{diag}\left\{Q_{i}, 0\right\}$. Note that $Q_{i}>0$ and $R_{i}>0$. By Lemma 3.1, it is obviously shown from inequalities (3.20), (3.21), and the second inequalities of (3.15) and (3.16) that the closedloop system (3.11) is stable.

In addition, it can be seen from inequalities (3.20) and (3.21) that

$$
\begin{aligned}
\left(\bar{A}_{i}+\Delta \bar{A}_{i}-\left(\bar{B}_{i}+\Delta \bar{B}_{i}\right) \bar{L}_{i}\right)^{T} \bar{P}_{i}+\bar{P}_{i}\left(\bar{A}_{i}+\Delta \bar{A}_{i}-\left(\bar{B}_{i}+\Delta \bar{B}_{i}\right) \bar{L}_{i}\right) \\
+\bar{E}_{i}^{T} U_{i} \bar{E}_{i}+\bar{Q}_{i}+\bar{L}_{i}^{T} R_{i} \bar{L}_{i} \leq 0, \quad i \in \mathbb{I} .
\end{aligned}
$$

Multiplying from left and right by $\overline{\mathbf{x}}^{T}$ and $\overline{\mathbf{x}}$, respectively, and removing the nonnegative term $\overline{\mathbf{x}}^{T} \bar{E}_{i}^{T} U_{i} \bar{E}_{i} \overline{\mathbf{x}}$ render

$$
\frac{\mathrm{d}}{\mathrm{d} t}\left(\overline{\mathbf{x}}^{T} \bar{P}_{i} \overline{\mathbf{x}}\right)+\overline{\mathbf{x}}^{T} \bar{Q}_{i} \overline{\mathbf{x}}+\mathbf{u}^{T} R_{i} \mathbf{u} \geq 0
$$

Integration from 0 to $\infty$, and noticing the global stability of closed-loop system (3.11), gives the result of (3.18). The proof is thus completed.

It is shown that the matrix inequalities (3.15) and (3.16) are BMIs due to the bilinear forms of $\bar{P}_{i} \bar{B}_{i} \bar{L}_{i}$ and $\varepsilon_{i} \bar{L}_{i}$ when both the Lyapunov matrix $\bar{P}_{i}$ and the feedback gain matrix $\bar{L}_{i}$ become the variables to be determined. Our interest is to find a parameter matrix $L$ to minimize the upper bound $\overline{\mathbf{x}}_{0}^{T} \bar{P}_{i_{0}} \overline{\mathbf{x}}_{0}$ on the cost function (3.5) for the state feedback closedloop system (3.11). Then, the optimization problem can be formulated as

$$
\begin{gathered}
\min _{L, S, U_{i}, W_{i}, \varepsilon_{i}} \overline{\mathbf{x}}_{0}^{T} \bar{P}_{i_{0}} \overline{\mathbf{x}}_{0} \\
\text { s.t. }\left\{\begin{array}{l}
\bar{L}_{i} \in \mathbb{L} \\
(3.15)-(3.16),
\end{array}\right.
\end{gathered}
$$

where $i \in \mathbb{I}$, and $\mathbb{L}$ is the set of admissible values for the state feedback gain matrix $\bar{L}_{i}$, bounded by practical design constraints.

Remark 3.4. It should be noted that the optimization problem (3.24) is a nonconvex optimization problem with the BMIs constraints of (3.15) and (3.16). For BMIs problem, we [28] recently have already designed a mixed algorithm combining genetic algorithm (GA) and interior point method to solve it. Here, we can use the mixed algorithm proposed in [28] to obtain the optimal controller parameter matrix $L$ and the corresponding objective $\overline{\mathbf{x}}_{0}^{T} \bar{P}_{i_{0}} \overline{\mathbf{x}}_{0}$. 
In general, one can set the parameter matrix $\mathrm{L}$ to be the decision variables searched by GA. For a given chromosome corresponding to $\mathrm{L}$, the nonconvex problem (3.24) reduces to an SDP involving LMIs which can be solved efficiently by Matlab LMI toolbox.

Remark 3.5. It should be pointed out that when solving the BMIs problem which is an NP hard problem in essence, the mixed algorithm combining GA with the interior point method may suffer from long computational time, especially for high-dimensional systems. Therefore, the optimal control problem can only be solved offline. In addition, the approximation error introduced by the linearization procedure for the chaotic system in Section 2 may adversely impact the stability analysis of the closed-loop system. To overcome this negative impact, one can divide the state space into a more sophisticated partition, but this will also increase the computational burden. Thus, one should seek a balance between the solution accuracy and the computational burden. On the other hand, for the chaotic systems there exists at least a bounded attractor. Due to the boundedness of the chaotic attractor, a relatively fine partition can be achieved to reduce the approximation error in the piecewise linearization procedure, which leads to a controller with a good performance.

To tell if the solutions obtained above are close to optimality or not, we must set up a lower bound on cost function (3.5). The result is presented as follows.

Theorem 3.6. If there exist a set of constants $\varepsilon_{i}>0$ and symmetric matrices $S$ and $U_{i}$ such that $U_{i}$ have nonnegative entries, while $P_{i}=F_{i}^{T} S F_{i}, i \in \mathbb{I}_{0}$ and $\bar{P}_{i}=\bar{F}_{i}^{T} S \bar{F}_{i}, i \in \mathbb{I}_{1}$ satisfy

$$
\left[\begin{array}{ccc}
\Psi_{i} & P_{i} B_{i}-\varepsilon_{i} N_{A_{i}}^{T} N_{B_{i}} & P_{i} M_{i} \\
B_{i}^{T} P_{i}-\varepsilon_{i} N_{B_{i}}^{T} N_{A_{i}} & R_{i}-\varepsilon_{i} N_{B_{i}}^{T} N_{B_{i}} & 0 \\
M_{i}^{T} P_{i} & 0 & \varepsilon_{i} I
\end{array}\right]>0,
$$

for $i \in \mathbb{I}_{0}$,

$$
\left[\begin{array}{ccc}
\bar{\Psi}_{i} & \bar{P}_{i} \bar{B}_{i}-\varepsilon_{i} \bar{N}_{A_{i}}^{T} N_{B_{i}} & \bar{P}_{i} \bar{M}_{i} \\
\bar{B}_{i}^{T} \bar{P}_{i}-\varepsilon_{i} N_{B_{i}}^{T} \bar{N}_{A_{i}} & R_{i}-\varepsilon_{i} N_{B_{i}}^{T} N_{B_{i}} & 0 \\
\bar{M}_{i}^{T} \bar{P}_{i} & 0 & \varepsilon_{i} I
\end{array}\right]>0,
$$

for $i \in \mathbb{I}_{1}$, where

$$
\begin{gathered}
\Psi_{i}:=A_{i}^{T} P_{i}+P_{i} A_{i}+Q_{i}-E_{i}^{T} U_{i} E_{i}-\varepsilon_{i} N_{A_{i}}^{T} N_{A_{i}} \\
\bar{\Psi}_{i}:=\bar{A}_{i}^{T} \bar{P}_{i}+\bar{P}_{i} \bar{A}_{i}+\bar{Q}_{i}-\bar{E}_{i}^{T} U_{i} \bar{E}_{i}-\varepsilon_{i} \bar{N}_{A_{i}}^{T} \bar{N}_{A_{i}},
\end{gathered}
$$

then for every trajectory $\mathbf{x}(t)$ of the uncertain system (3.4) with $\mathbf{x}(\infty)=0, \mathbf{x}(0)=\mathbf{x}_{0} \in X_{i_{0}}$, the cost function (3.5) satisfies

$$
J \geq \sup _{S, U_{i}, \varepsilon_{i}} \overline{\mathbf{x}}_{0}^{T} \bar{P}_{i_{0}} \overline{\mathbf{x}}_{0}
$$


Proof. We will first show the conditions for the cost function (3.5) satisfying the lower bound (3.28) can be guaranteed by

$$
\left[\begin{array}{cc}
\left(A_{i}+\Delta A_{i}\right)^{T} P_{i}+P_{i}\left(A_{i}+\Delta A_{i}\right)+Q_{i}-E_{i}^{T} U_{i} E_{i} & P_{i}\left(B_{i}+\Delta B_{i}\right) \\
\left(B_{i}+\Delta B_{i}\right)^{T} P_{i} & R_{i}
\end{array}\right]>0,
$$

for $i \in \mathbb{I}_{0}$, and

$$
\left[\begin{array}{cc}
\left(\bar{A}_{i}+\Delta \bar{A}_{i}\right)^{T} \bar{P}_{i}+\bar{P}_{i}\left(\bar{A}_{i}+\Delta \bar{A}_{i}\right)+\bar{Q}_{i}-\bar{E}_{i}^{T} U_{i} \bar{E}_{i} & \bar{P}_{i}\left(\bar{B}_{i}+\Delta \bar{B}_{i}\right) \\
\left(\bar{B}_{i}+\Delta \bar{B}_{i}\right)^{T} \bar{P}_{i} & R_{i}
\end{array}\right]>0,
$$

for $i \in \mathbb{I}_{1}$.

Actually, for $i \in \mathbb{I}$, we can get from (3.29) and (3.30) that

$$
\left[\begin{array}{cc}
\left(\bar{A}_{i}+\Delta \bar{A}_{i}\right)^{T} \bar{P}_{i}+\bar{P}_{i}\left(\bar{A}_{i}+\Delta \bar{A}_{i}\right)+\bar{Q}_{i}-\bar{E}_{i}^{T} U_{i} \bar{E}_{i} & \bar{P}_{i}\left(\bar{B}_{i}+\Delta \bar{B}_{i}\right) \\
\left(\bar{B}_{i}+\Delta \bar{B}_{i}\right)^{T} \bar{P}_{i} & R_{i}
\end{array}\right] \geq 0
$$

Multiplying from left and right by $\left[\overline{\mathbf{x}}^{T}, \mathbf{u}^{T}\right]$ and $\left[\overline{\mathbf{x}}^{T}, \mathbf{u}^{T}\right]^{T}$, respectively, and removing the nonnegative term $\overline{\mathbf{x}}^{T} \bar{E}_{i}^{T} U_{i} \bar{E}_{i} \overline{\mathbf{x}}$ yield

$$
\begin{aligned}
0 & \leq 2 \overline{\mathbf{x}}^{T} \bar{P}_{i}\left(\left(\bar{A}_{i}+\Delta \bar{A}_{i}\right) \overline{\mathbf{x}}+\left(\bar{B}_{i}+\Delta \bar{B}_{i}\right) \mathbf{u}\right)+\overline{\mathbf{x}}^{T} \bar{Q}_{i} \overline{\mathbf{x}}+\mathbf{u}^{T} R_{i} \mathbf{u} \\
& =\frac{\mathrm{d}}{\mathrm{d} t}\left(\overline{\mathbf{x}}^{T} \bar{P}_{i} \overline{\mathbf{x}}\right)+\overline{\mathbf{x}}^{T} \bar{Q}_{i} \overline{\mathbf{x}}+\mathbf{u}^{T} R_{i} \mathbf{u}
\end{aligned}
$$

Integration from 0 to $\infty$, and noticing $\mathbf{x}(\infty)=0$, gives the result of (3.28).

Next, we will show that inequality (3.29) is equivalent to (3.25). For simplifying the presentation, denote

$$
G:=\left[\begin{array}{cc}
A_{i}^{T} P_{i}+P_{i} A_{i}+Q_{i}-E_{i}^{T} U_{i} E_{i} & P_{i} B_{i} \\
B_{i}^{T} P_{i} & R_{i}
\end{array}\right]
$$

Note the uncertain form (3.2). Then, inequality (3.29) can be written as

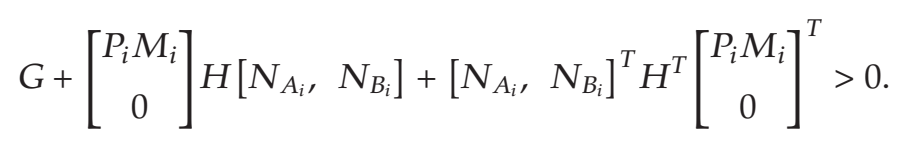

By Lemma 3.2, inequality (3.34) is equivalent to the existence of some $\varepsilon_{i}>0$ such that

$$
G-\varepsilon_{i}^{-1}\left[\begin{array}{c}
P_{i} M_{i} \\
0
\end{array}\right]\left[\begin{array}{c}
P_{i} M_{i} \\
0
\end{array}\right]^{T}-\varepsilon_{i}\left[\begin{array}{ll}
N_{A_{i}}, & N_{B_{i}}
\end{array}\right]^{T}\left[\begin{array}{ll}
N_{A_{i}}, & N_{B_{i}}
\end{array}\right]>0
$$


that is,

$$
\left[\begin{array}{cr}
A_{i}^{T} P_{i}+P_{i} A_{i}+Q_{i}-E_{i}^{T} U_{i} E_{i}-\varepsilon_{i} N_{A_{i}}^{T} N_{A_{i}}-\varepsilon_{i}^{-1} P_{i} M_{i} M_{i}^{T} P_{i} & P_{i} B_{i}-\varepsilon_{i} N_{A_{i}}^{T} N_{B_{i}} \\
B_{i}^{T} P_{i}-\varepsilon_{i} N_{B_{i}}^{T} N_{A_{i}} & R_{i}-\varepsilon_{i} N_{B_{i}}^{T} N_{B_{i}}
\end{array}\right]>0
$$

which, by Schur complement, is equivalent to inequality (3.25). By similar techniques, it can also be shown that inequality (3.30) is equivalent to inequality (3.26). The proof is complete.

Remark 3.7. It is shown that inequalities (3.25) and (3.26) are LMIs about the variables $P_{i}, \bar{P}_{i}$ and $\varepsilon_{i}$. So the problem of maximizing the lower bound (3.28) can be cast as an SDP with LMIs constraints of (3.25) and (3.26), and solved numerically effectively.

Remark 3.8. In the above analysis, it is assumed that the initial condition $\mathbf{x}_{0}$ is given or known in advance. Note that the bounds in (3.18) and (3.28) depend on the initial state $\mathbf{x}_{0}$. To remove this dependence on the initial state, we can use the techniques developed in [28] and extend the corresponding results to the case where the initial condition $\mathbf{x}_{0}$ is a random variable subjected to uniform distribution on a certain bounded region $X_{0}$. For further details, please refer to $[28]$.

The global quadratic Lyapunov function technique is often applied in the control synthesis of dynamical systems [26]. In the following, by virtue of the global quadratic Lyapunov function technique and linear feedback control law, we present an optimal guaranteed cost control method for the chaotic system (2.1) associated with the cost function (2.2), which with the comparisons in the simulation results will show advantages of the obtained results in Theorems 3.3 and 3.6.

Consider the following linear feedback control law:

$$
\mathbf{u}=-\tilde{L} \mathbf{x}
$$

Substituting the control law (3.37) into system (2.1), we can get the following closed-loop system:

$$
\dot{\mathbf{x}}(t)=(A-B \tilde{L}) \mathbf{x}(t)+F(\mathbf{x})
$$

Additionally, note the boundedness of the chaotic attractor and the Lipschitz continuity condition for the nonlinear term $F(\mathbf{x})$. There exist some matrix $\Gamma \geq 0$ and a bounded set $\Omega$ which bounds the chaotic attractor, such that

$$
F^{T}(\mathbf{x}) F(\mathbf{x}) \leq \mathbf{x}^{T} \Gamma^{2} \mathbf{x}, \quad \forall \mathbf{x} \in \Omega .
$$

The upper bound on the cost function (2.2) for the chaotic system (2.1) by applying linear feedback control law (3.37) is presented as follows. 
Theorem 3.9. Consider system (2.1) with the initial condition $\mathbf{x}_{0} \in \Omega$. If there exist positive constants $\alpha, \beta$, positive definite matrix $Y$, and any matrix $Z$ with appropriate dimensions such that

$$
\begin{aligned}
& {\left[\begin{array}{cccc}
A Y+Y A^{T}-B Z-Z^{T} B^{T}+\alpha I & Y \Gamma & Y & Z^{T} \\
\Gamma Y & -\alpha I & 0 & 0 \\
Y & 0 & -Q^{-1} & 0 \\
Z & 0 & 0 & -R^{-1}
\end{array}\right]<0,} \\
& {\left[\begin{array}{cc}
-\beta & \mathbf{x}_{0}^{T} \\
\mathbf{x}_{0} & -Y
\end{array}\right]<0,}
\end{aligned}
$$

then the closed-loop system (3.38) is globally exponentially stable, and the cost function (2.2) satisfies

$$
J<\beta .
$$

Furthermore, the corresponding control law can be obtained as $\mathbf{u}=-Z Y^{-1} \mathbf{x}$.

Proof. Denote $P=Y^{-1}>0$. Construct the Lyapunov function candidate as

$$
V(\mathbf{x})=\mathbf{x}^{T} P \mathbf{x}
$$

By virtue of the fact that $M^{T} N+N^{T} M \leq \alpha^{-1} M^{T} M+\alpha N^{T} N$, for all $\alpha>0$, and matrices $M$ and $N$ with appropriate dimensions, calculating the time derivative of $V(\mathbf{x})$ along the trajectory of the closed-loop system (3.38) and noticing (3.39) yield

$$
\begin{aligned}
\frac{\mathrm{d} V(\mathbf{x})}{\mathrm{d} t} & =\mathbf{x}^{T}\left((A-B \tilde{L})^{T} P+P(A-B \tilde{L})\right) \mathbf{x}+F^{T}(\mathbf{x}) P \mathbf{x}+\mathbf{x}^{T} P F(\mathbf{x}) \\
& \leq \mathbf{x}^{T}\left((A-B \tilde{L})^{T} P+P(A-B \tilde{L})+\alpha P^{2}\right) \mathbf{x}+\alpha^{-1} F^{T}(\mathbf{x}) F(\mathbf{x}) \\
& \leq \mathbf{x}^{T}\left((A-B \tilde{L})^{T} P+P(A-B \tilde{L})+\alpha P^{2}+\alpha^{-1} \Gamma^{2}\right) \mathbf{x} .
\end{aligned}
$$

On the other hand, by Schur complement, the first inequality of (3.40) is equivalent to

$$
A Y+Y A^{T}-B Z-Z^{T} B^{T}+\alpha I+\alpha^{-1} Y \Gamma^{2} Y+Y Q Y+Z^{T} R Z<0
$$

Noticing $Y P=I, \widetilde{L}=Z P$, pre- and postmultiplying both sides of (3.44) by $P$ implies

$$
(A-B \tilde{L})^{T} P+P(A-B \tilde{L})+\alpha P^{2}+\alpha^{-1} \Gamma^{2}+Q+\tilde{L}^{T} R \tilde{L}<0
$$


Thus, it follows from (3.43) and (3.45) that

$$
\frac{\mathrm{d} V(\mathbf{x})}{\mathrm{d} t}+\mathbf{x}^{T} Q \mathbf{x}+\mathbf{x}^{T} \tilde{L}^{T} R \tilde{L} \mathbf{x}<0 .
$$

Note that $Q>0$ and $R>0$. It is obvious that $\mathrm{d} V(\mathbf{x}) / \mathrm{d} t<0$ which guarantees the global stability of closed-loop system (3.38), that is, $\mathbf{x}(\infty)=0$.

Integration both sides of (3.46) from 0 to $\infty$, and noticing $V(\mathbf{x}(\infty))=0$, renders

$$
J<V\left(\mathbf{x}_{0}\right)=\mathbf{x}_{0} Y^{-1} \mathbf{x}_{0}
$$

with which combining the second inequality of (3.40) shows the result of (3.41). The proof is complete.

Remark 3.10. It is shown that the inequalities in (3.40) are LMIs in the variables $Y, Z, \alpha$, $\beta$. So the problem of minimizing the upper bound (3.41) can be cast as an SDP with LMIs constraints of (3.40) and can be solved numerically effectively. On the other hand, it should be pointed out that the control synthesis methods based on the global quadratic Lyapunov function (3.42) and linear feedback control law (3.37) are conservative in practice compared with those in Theorems 3.3 and 3.6, which will be shown in illustrative examples.

\section{Illustrative Examples}

In this section, we will give two examples to illustrate the effectiveness of the proposed methods.

\subsection{Genesio-Tesi Chaotic System}

Consider the Genesio-Tesi chaotic system presented in [23], and the controlled system is described as follows:

$$
\left[\begin{array}{l}
\dot{x}_{1} \\
\dot{x}_{2} \\
\dot{x}_{3}
\end{array}\right]=\left[\begin{array}{ccc}
0 & 1 & 0 \\
0 & 0 & 1 \\
-p_{1} & -p_{2} & -p_{3}
\end{array}\right]\left[\begin{array}{l}
x_{1} \\
x_{2} \\
x_{3}
\end{array}\right]+\left[\begin{array}{l}
0 \\
0 \\
x_{1}^{2}
\end{array}\right]+\left[\begin{array}{l}
1 \\
1 \\
1
\end{array}\right] u,
$$

where $p_{1}=6, p_{2}=2.92, p_{3}=1.2$.

Denote that $c^{T}=[1,0,0]$ and $\mathbf{x}^{T}=\left[x_{1}, x_{2}, x_{3}\right]$. Note the boundedness of the chaotic attractor shown in [23]. The state space can be confined to $X:=\left\{\mathbf{x} \mid-6 \leq c^{T} \mathbf{x} \leq 6\right\}$ by simulation. The partition of state space is set to be

$$
\begin{gathered}
X_{1}=\left\{\mathbf{x} \mid c^{T} \mathbf{x} \in[-1,1)\right\}, \quad X_{2}=\left\{\mathbf{x} \mid c^{T} \mathbf{x} \in[1,3)\right\}, \quad X_{3}=\left\{\mathbf{x} \mid c^{T} \mathbf{x} \in[3,6]\right\}, \\
X_{4}=\left\{\mathbf{x} \mid c^{T} \mathbf{x} \in[-3,-1)\right\}, \quad X_{5}=\left\{\mathbf{x} \mid c^{T} \mathbf{x} \in[-6,-3)\right\} .
\end{gathered}
$$


Then, the nonlinear term $x_{1}^{2}$ can be described as

$$
x_{1}^{2}=k_{i} x_{1}+l_{i}+\delta_{i}\left(x_{1}\right), \quad \mathbf{x} \in X_{i}, i=1,2,3,4,5,
$$

where $\delta_{i}$ denotes the approximation error. Taking $k_{1}=0, k_{2}=-k_{4}=4.5, k_{3}=-k_{5}=9, l_{1}=0$, $l_{2}=l_{4}=-4.5, l_{3}=l_{5}=-18$, one can obtain that

$$
\begin{gathered}
\left|\delta_{1}\left(x_{1}\right)\right| \leq\left|x_{1}\right|, \quad\left|\delta_{2}\left(x_{1}\right)\right| \leq 1, \quad\left|\delta_{3}\left(x_{1}\right)\right| \leq 2.25, \\
\left|\delta_{4}\left(x_{1}\right)\right| \leq 1, \quad\left|\delta_{5}\left(x_{1}\right)\right| \leq 2.25 .
\end{gathered}
$$

Note the expressions (4.3) and (4.4). System (4.1) can be converted to the piecewise linear system (3.1) with

$$
\begin{aligned}
& A_{1}=\left[\begin{array}{ccc}
0 & 1 & 0 \\
0 & 0 & 1 \\
-6 & -2.92 & -1.2
\end{array}\right], \quad A_{2}=\left[\begin{array}{ccc}
0 & 1 & 0 \\
0 & 0 & 1 \\
-1.5 & -2.92 & -1.2
\end{array}\right], \quad A_{3}=\left[\begin{array}{ccc}
0 & 1 & 0 \\
0 & 0 & 1 \\
3 & -2.92 & -1.2
\end{array}\right], \\
& A_{4}=\left[\begin{array}{ccc}
0 & 1 & 0 \\
0 & 0 & 1 \\
-10.5 & -2.92 & -1.2
\end{array}\right], \quad A_{5}=\left[\begin{array}{ccc}
0 & 1 & 0 \\
0 & 0 & 1 \\
-15 & -2.92 & -1.2
\end{array}\right], \quad B_{i}=\left[\begin{array}{l}
1 \\
1 \\
1
\end{array}\right] \text {, } \\
& a_{1}=0, \quad a_{2}=a_{4}=\left[\begin{array}{lll}
0 & 0 & -4.5
\end{array}\right]^{T}, \quad a_{3}=a_{5}=\left[\begin{array}{lll}
0 & 0 & -18
\end{array}\right]^{T}, \\
& \Delta A_{1}=M_{1} H N_{A_{1}}, \quad \Delta A_{2}=\Delta A_{3}=\Delta A_{4}=\Delta A_{5}=0, \quad \Delta B_{i}=0, \\
& \Delta a_{1}=0, \quad \Delta a_{2}=M_{2} H N_{a_{2}}, \quad \Delta a_{3}=M_{3} H N_{a_{3}}, \\
& \Delta a_{4}=M_{4} H N_{a_{4}}, \quad \Delta a_{5}=M_{5} H N_{a_{5}}, \\
& M_{i}=\left[\begin{array}{ll}
0 & \\
& 0 \\
& 2
\end{array}\right], \quad N_{A_{1}}=\left[\begin{array}{ccc}
0 & 0 & 0 \\
0 & 0 & 0 \\
0.5 & 0 & 0
\end{array}\right], \\
& N_{a_{2}}=N_{a_{4}}=\left[\begin{array}{c}
0 \\
0 \\
0.5
\end{array}\right], \quad N_{a_{3}}=N_{a_{5}}=\left[\begin{array}{c}
0 \\
0 \\
1.125
\end{array}\right] \text {, }
\end{aligned}
$$

where $i=1, \ldots, 5$, and $H$ is an uncertain matrix bounded by $H^{T} H \leq I$.

It is worthwhile to mention that the nominal autonomous piecewise linear system (3.1) with parameters (4.5), that is, $u \equiv 0, \Delta A_{i}=0, \Delta a_{i}=0$, can exhibit chaotic dynamics, and the strange attractor is depicted in Figure 1. It is shown from Figure 1 that the system (3.1) with parameters (4.5) evolves to a single-scroll chaotic attractor, which is similar to the GenesioTesi chaotic attractor. Thus, it is indicated that the piecewise linear system approximating a chaotic system can preserve the complex dynamic behaviors of the original system. 


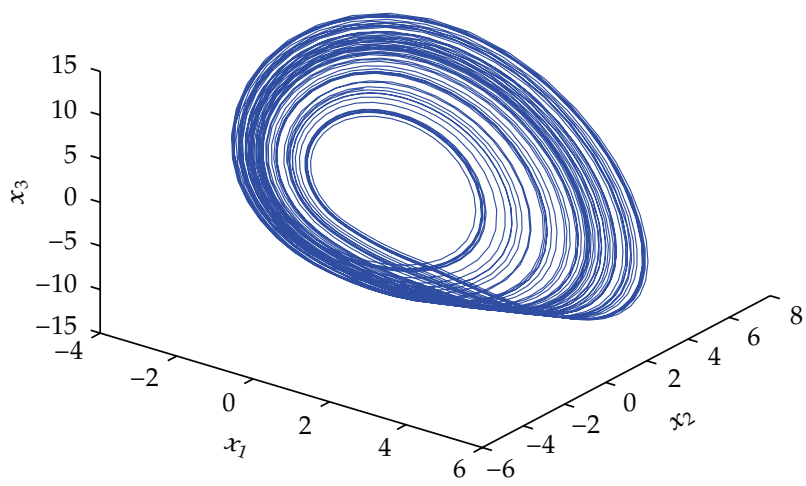

Figure 1: Phase portraits of the nominal autonomous system (3.1) with parameters (4.5).

Consider the cost function (2.2) with $Q=\operatorname{diag}\{1,1,1\}, R=1$, and the initial value $\mathbf{x}_{0}=$ $[-1.8,-1,1]^{T}$ of system (4.1). The matrices $\bar{E}_{i}$ and $\bar{F}_{i}$ can be constructed by virtue of the method proposed in [20]. Assume that the feedback gain matrix $\bar{L}_{i}$ is bounded by $\left\|\bar{L}_{i}\right\|_{\infty}<12$, where $\left\|\bar{L}_{i}\right\|_{\infty}$ denotes the largest absolute value among all the entries of vector $\bar{L}_{i}$. Then, applying the mixed algorithm provided in [28], we solve the BMIs problem (3.24) based on Theorem 3.3 with the code written in MATLAB 7.0 and get the optimal upper bound on $J$, denoted as $\bar{J}^{*}$, and the corresponding optimal parameter matrix $L^{*}$ as follows:

$$
\begin{gathered}
\bar{J}^{*}=17.7528, \\
L^{*}=[-3.3088,-0.3345,2.7111,1.2363,-0.0548]^{T} .
\end{gathered}
$$

According to the expression of (3.10), we can get the following state feedback gain matrices:

$$
\begin{gathered}
L_{1}=[2.7111,1.2363,-0.0548], \\
\bar{L}_{2}=[2.3765,1.2363,-0.0548,0.3345], \\
\bar{L}_{3}=[6.0198,1.2363,-0.0548,-10.5954], \\
\bar{L}_{4}=[6.0198,1.2363,-0.0548,3.3088], \\
\bar{L}_{5}=[2.3765,1.2363,-0.0548,-7.6212],
\end{gathered}
$$

with which the optimal control $u$ taking the form of piecewise linear feedback control law (3.9) can be obtained.

Actually, the cost function (2.2) for the closed-loop system (4.1) with above controller gain matrices is computed as $J=13.1623$. The numerical simulation of system (4.1) with the piecewise linear state feedback control is shown in Figure 2.

In addition, according to Theorem 3.6, the maximal lower bound on $J$, denoted as $J^{*}$, can be obtained by solving the corresponding SDP with the LMI toolbox in MATLAB 7.0 as follows:

$$
\underline{J}^{*}=10.2047
$$




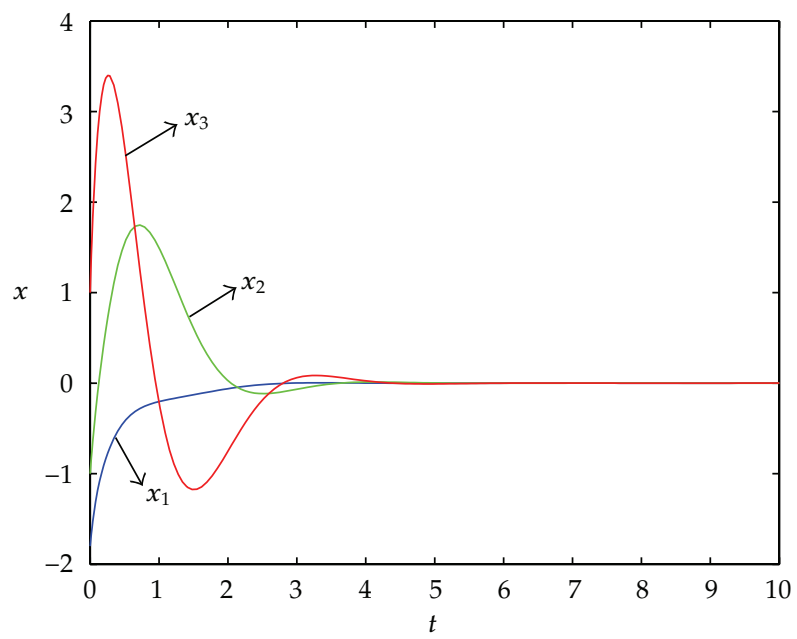

Figure 2: Time response of system (4.1) with the piecewise linear state feedback.

On the other hand, note that $-6 \leq c^{T} \mathbf{x} \leq 6$. The matrix $\Gamma$ in (3.39) can be obtained as $\Gamma=\operatorname{diag}\{6,0,0\}$. According to Theorem 3.9, we solve the corresponding SDP, and obtain the optimal gain matrix $\widetilde{L}^{*}$ in (3.37) and upper bound $\beta^{*}$ as follows:

$$
\widetilde{L}^{*}=Z Y^{-1}=[14.1843,1.1514,-0.3980], \quad \beta^{*}=53.0699,
$$

which shows a fact that the optimal control methods based on the global quadratic Lyapunov function are conservative compared with those in Theorem 3.3.

\subsection{A New Chaotic System}

Consider the new chaotic system presented in [26], and the controlled system is described as follows:

$$
\left[\begin{array}{c}
\dot{x}_{1} \\
\dot{x}_{2} \\
\dot{x}_{3}
\end{array}\right]=\left[\begin{array}{ccc}
0 & 1 & 0 \\
0 & 0 & 1 \\
-p_{1} & -p_{1} & -p_{1}
\end{array}\right]\left[\begin{array}{l}
x_{1} \\
x_{2} \\
x_{3}
\end{array}\right]+\left[\begin{array}{c}
0 \\
0 \\
p_{2} \tanh \left(x_{1}\right)
\end{array}\right]+\left[\begin{array}{c}
0.5 \\
1 \\
1
\end{array}\right] u
$$

where $p_{1}=0.5, p_{2}=5$, and the hyperbolic function $\tanh (x)=(\exp (x)-\exp (-x)) /(\exp (x)+$ $\exp (-x)$ ). The strange attractor of the autonomous system (4.10) with $u \equiv 0$ is shown in Figure 3, which is a double-scroll chaotic attractor. 


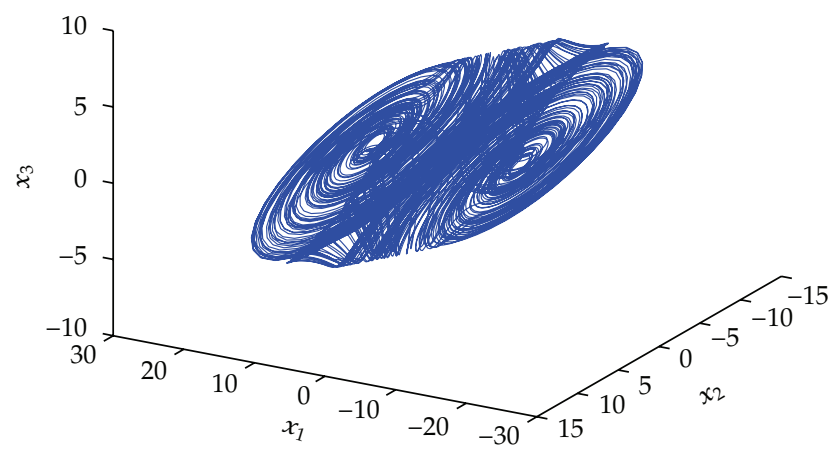

Figure 3: Phase portraits of the autonomous system (4.10).

Note the boundedness of the chaotic attractor shown in Figure 3. The state space can be confined to $X:=\left\{\mathbf{x} \mid-23.3 \leq c^{T} \mathbf{x} \leq 23.3\right\}$ by simulation. The partition of state space is set to be

$$
\begin{gathered}
X_{1}=\left\{\mathbf{x} \mid c^{T} \mathbf{x} \in[-23.3,-1.18)\right\}, \quad X_{2}=\left\{\mathbf{x} \mid c^{T} \mathbf{x} \in[-1.18,1.18)\right\}, \\
X_{3}=\left\{\mathbf{x} \mid c^{T} \mathbf{x} \in[1.18,23.3]\right\} .
\end{gathered}
$$

Then, the nonlinear term $\tanh \left(x_{1}\right)$ can be described as

$$
\tanh \left(x_{1}\right)= \begin{cases}k_{1} x_{1}+l_{1}+\delta_{1}\left(x_{1}\right), & \mathbf{x} \in X_{1} \\ k_{2} x_{1}+l_{2}+\delta_{2}\left(x_{1}\right), & \mathbf{x} \in X_{2} \\ k_{3} x_{1}+l_{3}+\delta_{3}\left(x_{1}\right), & \mathbf{x} \in X_{3}\end{cases}
$$

where $\delta_{i}$ denotes the approximation error. Taking $k_{1}=0, k_{2}=0.85, k_{3}=0, l_{1}=-1, l_{2}=0$, $l_{3}=1$, one can obtain that

$$
\left|\delta_{1}\left(x_{1}\right)\right| \leq 0.17, \quad\left|\delta_{2}\left(x_{1}\right)\right| \leq 0.15\left|x_{1}\right|, \quad\left|\delta_{3}\left(x_{1}\right)\right| \leq 0.17
$$

Note the expressions (4.12) and (4.13). System (4.10) can be converted to the piecewise linear system (3.1) with

$$
\begin{gathered}
A_{1}=A_{3}=\left[\begin{array}{ccc}
0 & 1 & 0 \\
0 & 0 & 1 \\
-p_{1} & -p_{1} & -p_{1}
\end{array}\right], \quad A_{2}=\left[\begin{array}{ccc}
0 & 1 & 0 \\
0 & 0 & 1 \\
-p_{1}+0.85 p_{2} & -p_{1} & -p_{1}
\end{array}\right], \\
B_{1}=B_{2}=B_{3}=\left[\begin{array}{c}
0.5 \\
1 \\
1
\end{array}\right], \quad a_{1}=-a_{3}=\left[\begin{array}{c}
0 \\
0 \\
-p_{2}
\end{array}\right], \quad a_{2}=0
\end{gathered}
$$




$$
\begin{gathered}
\Delta A_{1}=\Delta A_{3}=0, \quad \Delta A_{2}=M_{2} H N_{A_{2}}, \quad \Delta B_{1}=\Delta B_{2}=\Delta B_{3}=0, \\
\Delta a_{1}=M_{1} H N_{a_{1}}, \quad \Delta a_{2}=0, \quad \Delta a_{3}=M_{3} H N_{a_{3}}, \\
M_{1}=M_{2}=M_{3}=\left[\begin{array}{ccc}
0 & \\
& 0 & \\
& & \\
&
\end{array}\right], \quad N_{A_{2}}=\left[\begin{array}{ccc}
0 & 0 & 0 \\
0 & 0 & 0 \\
0.15 p_{2} & 0 & 0
\end{array}\right], \quad N_{a_{1}}=N_{a_{3}}=\left[\begin{array}{c}
0 \\
0 \\
0.17 p_{2}
\end{array}\right] .
\end{gathered}
$$

Consider the cost function (2.2) with $Q=\operatorname{diag}\{0.8,0.8,0.8\}, R=1.2$, and the system initial value $\mathbf{x}_{0}=[1.4,1,-0.6]^{T}$. Assume that the feedback gain matrix $\bar{L}_{i}$ is bounded by $\left\|\bar{L}_{i}\right\|_{\infty}<8$. Then, similarly to the above subsection, we get the maximal lower bound $J^{*}$, the optimal upper bound $\bar{J}^{*}$, and the corresponding optimal parameter matrix $L^{*}$ as follows:

$$
\begin{gathered}
J^{*}=5.5117, \quad \bar{J}^{*}=9.7024, \\
L^{*}=[-4.3655,1.2292,2.3555,1.3460,1.4357]^{T} .
\end{gathered}
$$

According to the expression of (3.10), we can get the following state feedback gain matrices:

$$
\begin{gathered}
\bar{L}_{1}=[6.7210,1.3460,1.4357,5.1513], \quad L_{2}=[2.3555,1.3460,1.4357], \\
\bar{L}_{3}=[3.5847,1.3460,1.4357,-1.4505]
\end{gathered}
$$

with which the optimal control $u$ taking the form of (3.9) is obtained.

Additionally, the cost function (2.2) for the closed-loop system (4.10) with above controller gain matrices is computed as $J=7.8725$. The numerical simulation of system (4.10) with piecewise linear state feedback control is shown in Figure 4.

Furthermore, note that $\tanh ^{2}\left(x_{1}\right) \leq x_{1}^{2}$. The matrix $\Gamma$ in (3.39) can be obtained as $\Gamma=$ $\operatorname{diag}\left\{p_{2}, 0,0\right\}$. According to Theorem 3.9, we solve the corresponding SDP, and obtain the optimal gain matrix $\tilde{L}^{*}$ in (3.37) and upper bound $\beta^{*}$ as follows:

$$
\widetilde{L}^{*}=Z Y^{-1}=[19.2415,2.5432,-0.2071], \quad \beta^{*}=98.965,
$$

which is significantly greater than the optimal upper bound $\bar{J}^{*}$ obtained from Theorem 3.3.

It is obviously shown from the above examples that the optimal upper bounds $\bar{J}^{*}$ obtained above get close to the corresponding lower bounds $J^{*}$, respectively. This implies that we have achieved or got close to the optimal control for the chaotic systems. Additionally, it should be pointed out that the newly reported chaotic system (4.10) is topologically not equivalent to the Genesio-Tesi chaotic system (4.1). However, by virtue of the optimal control methods proposed in this paper, both the different chaotic systems (4.1) and (4.10) can be optimally stabilized. The examples show the effectiveness of the proposed results. 


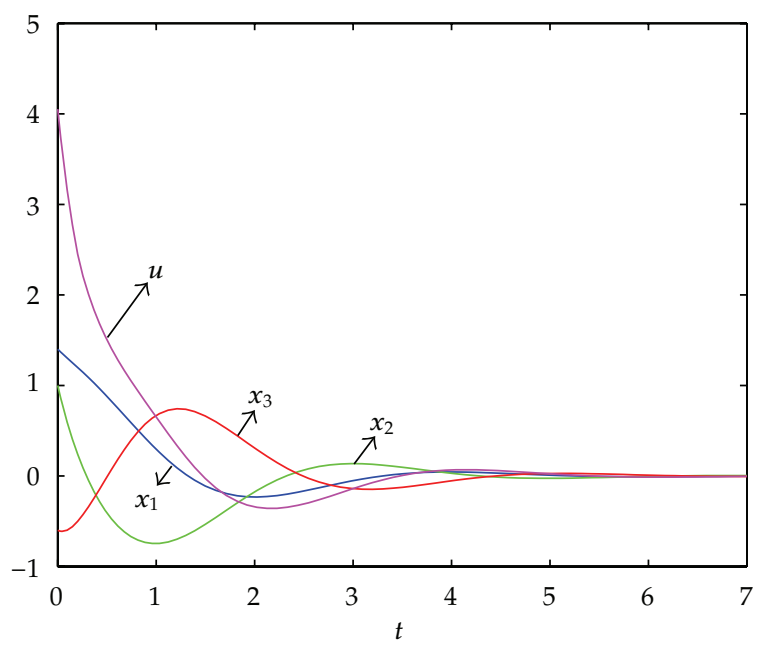

Figure 4: The control law and time response of the controlled system (4.10).

\section{Conclusion}

In this paper, we first convert a class of chaotic systems to the form of uncertain piecewise linear systems then investigate the optimal control for the chaotic systems where the piecewise linear state feedback optimal controller can be obtained by solving an optimization problem with BMIs constraints. The performance of the controller can be evaluated by the upper and lower bounds on the cost function. The optimal chaos synchronization for this class of chaotic systems will be studied in the near future.

\section{Acknowledgment}

The authors thank the anonymous referees and editor for their valuable comments and suggestions. This work was supported by the National Natural Science Foundation of China (no. 61004015), the Research Fund for the Doctoral Programme of Higher Education of China (no. 20090032120034), the Program for New Century Excellent Talents in Universities of China, and the Program for Changjiang Scholars and Innovative Research Team in University of China (no. IRT1028).

\section{References}

[1] G. Chen and X. Dong, From Chaos to Order, vol. 24 of Methodologies, Perspectives and Applications, World Scientific, Singapore, 1998.

[2] A. L. Fradkov and R. J. Evans, "Control of chaos: methods and applications in engineering," Annual Reviews in Control, vol. 29, no. 1, pp. 33-56, 2005.

[3] E. Ott, C. Grebogi, and J. A. Yorke, "Controlling chaos," Physical Review Letters, vol. 64, no. 11, pp. 1196-1199, 1990.

[4] C. Hernandez-Tenorio, T. L. Belyaeva, and V. N. Serkin, "Parametric resonance for solitons in the nonlinear Schrödinger equation model with time-dependent harmonic oscillator potential," Physica B, vol. 398, no. 2, pp. 460-463, 2007.

[5] H. Sun and H. Cao, "Chaos control and synchronization of a modified chaotic system," Chaos, Solitons and Fractals, vol. 37, no. 5, pp. 1442-1455, 2008. 
[6] G. Chen, "A simple adaptive feedback control method for chaos and hyper-chaos control," Applied Mathematics and Computation, vol. 217, no. 17, pp. 7258-7264, 2011.

[7] B. Niu and J. Wei, "Stability and bifurcation analysis in an amplitude equation with delayed feedback," Chaos, Solitons \& Fractals, vol. 37, no. 5, pp. 1362-1371, 2008.

[8] M. T. Yassen, "Chaos control of chaotic dynamical systems using backstepping design," Chaos, Solitons and Fractals, vol. 27, no. 2, pp. 537-548, 2006.

[9] M. S. Tavazoei, M. Haeri, and S. Jafari, "Fractional controller to stabilize fixed points of uncertain chaotic systems: Theoretical and experimental study," Proceedings of the Institution of Mechanical Engineers. Part I: Journal of Systems and Control Engineering, vol. 222, no. 3, pp. 175-184, 2008.

[10] J. M. Nazzal and A. N. Natsheh, "Chaos control using sliding-mode theory," Chaos, Solitons and Fractals, vol. 33, no. 2, pp. 695-702, 2007.

[11] M. Roopaei, B. Ranjbar Sahraei, and T.-C. Lin, "Adaptive sliding mode control in a novel class of chaotic systems," Communications in Nonlinear Science and Numerical Simulation, vol. 15, no. 12, pp. 4158-4170, 2010.

[12] W. J. Sun, “A global asymptotic synchronization problem via internal model approach," International Journal of Control, Automation, and Systems, vol. 8, no. 5, pp. 1153-1158, 2010.

[13] J. Wang, W. Tang, and J. Zhang, "Approximate synchronization of two non-linear systems via impulsive control," Proceedings of the Institution of Mechanical Engineers. Part I: Journal of Systems and Control Engineering, vol. 226, no. 13, pp. 338-347, 2012.

[14] X. R. Chen and C. X. Liu, "Chaos synchronization of fractional order unified chaotic system via nonlinear control," International Journal of Modern Physics B, vol. 25, pp. 407-415, 2011.

[15] A. Uçar, K. E. Lonngren, and E. W. Bai, "Synchronization of the unified chaotic systems via active control," Chaos, Solitons and Fractals, vol. 27, no. 5, pp. 1292-1297, 2006.

[16] Q. Jia, "Chaos control and synchronization of the Newton-Leipnik chaotic system," Chaos, Solitons and Fractals, vol. 35, no. 4, pp. 814-824, 2008.

[17] G. M. Mahmoud, T. Bountis, G. M. AbdEl-Latif, and E. E. Mahmoud, "Chaos synchronization of two different chaotic complex Chen and Lü systems," Nonlinear Dynamics, vol. 55, no. 1-2, pp. 43-53, 2009.

[18] K. Tanaka, T. Ikeda, and H. O. Wang, "A unified approach to controlling chaos via an LMI-based fuzzy control system design," IEEE Transactions on Circuits and Systems, vol. 45, no. 10, pp. 1021-1040, 1998.

[19] S. Boyd, L. El Ghaoui, E. Feron, and V. Balakrishnan, Linear Matrix Inequalities in System and Control Theory, vol. 15, Society for Industrial and Applied Mathematics SIAM, Philadelphia, Pa, USA, 1994.

[20] M. Johansson, Piecewise Linear Control Systems, vol. 284, Springer, Berlin, Germany, 2003.

[21] J. Zhang and W. Tang, "Analysis and control for a new chaotic system via piecewise linear feedback," Chaos, Solitons and Fractals, vol. 42, no. 4, pp. 2181-2190, 2009.

[22] M. Johansson and A. Rantzer, "Computation of piecewise quadratic Lyapunov functions for hybrid systems," IEEE Transactions on Automatic Control, vol. 43, no. 4, pp. 555-559, 1998.

[23] R. Genesio and A. Tesi, "Harmonic balance methods for the analysis of chaotic dynamics in nonlinear systems," Automatica, vol. 28, no. 3, pp. 531-548, 1992.

[24] A. Arneodo, P. Coullet, and C. Tresser, "A possible new mechanism for the onset of turbulence," Physics Letters A, vol. 81, no. 4, pp. 197-201, 1981.

[25] A. Tsuneda, "A gallery of attractors from smooth Chua's equation," International Journal of Bifurcation and Chaos, vol. 15, no. 1, pp. 1-49, 2005.

[26] J. Zhang and W. Tang, "Control and synchronization for a class of new chaotic systems via linear feedback," Nonlinear Dynamics, vol. 58, no. 4, pp. 675-686, 2009.

[27] L. Xie, "Output feedback $H_{\infty}$ control of systems with parameter uncertainty," International Journal of Control, vol. 63, no. 4, pp. 741-750, 1996.

[28] J. Zhang and W. Tang, "Output feedback optimal guaranteed cost control of uncertain piecewise linear systems," International Journal of Robust and Nonlinear Control, vol. 19, no. 5, pp. 569-590, 2009. 


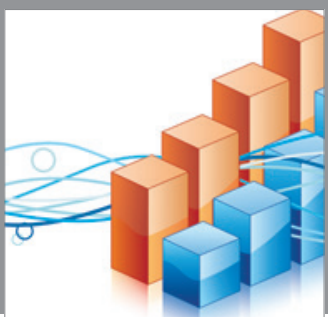

Advances in

Operations Research

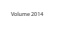

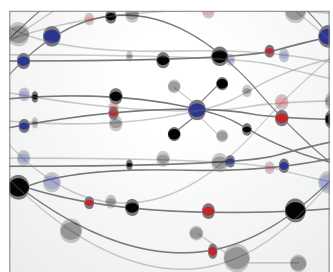

\section{The Scientific} World Journal
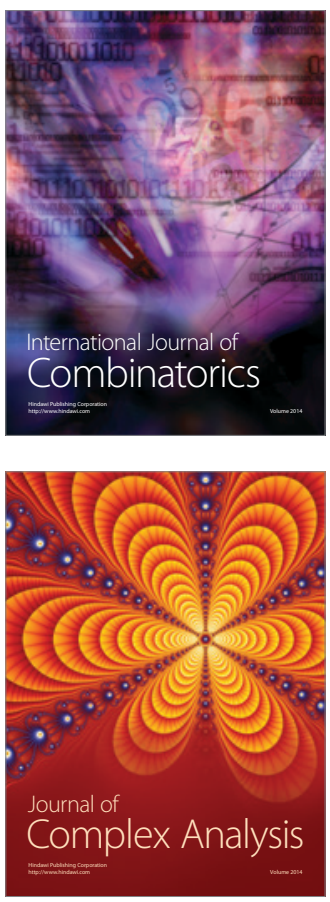

International Journal of

Mathematics and

Mathematical

Sciences
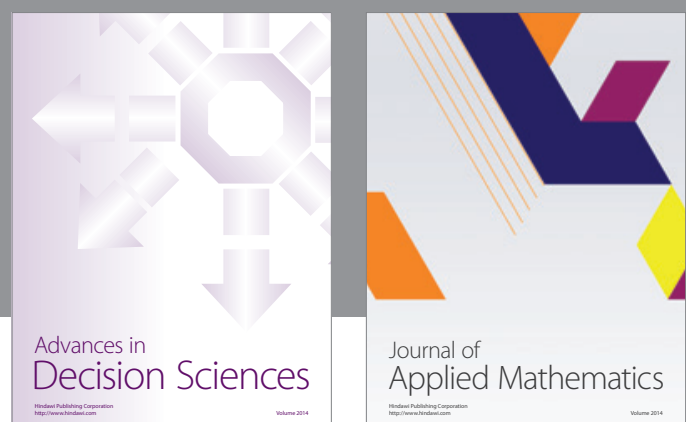

Journal of

Applied Mathematics
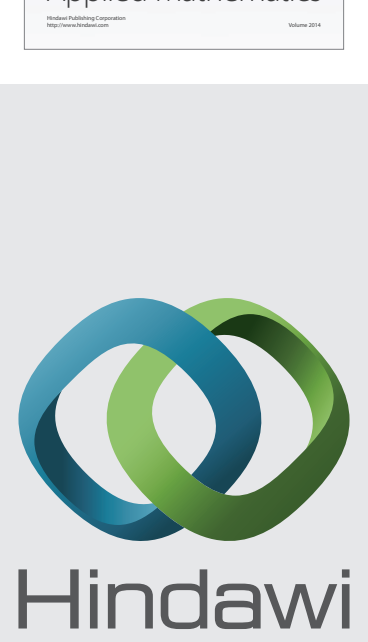

Submit your manuscripts at http://www.hindawi.com
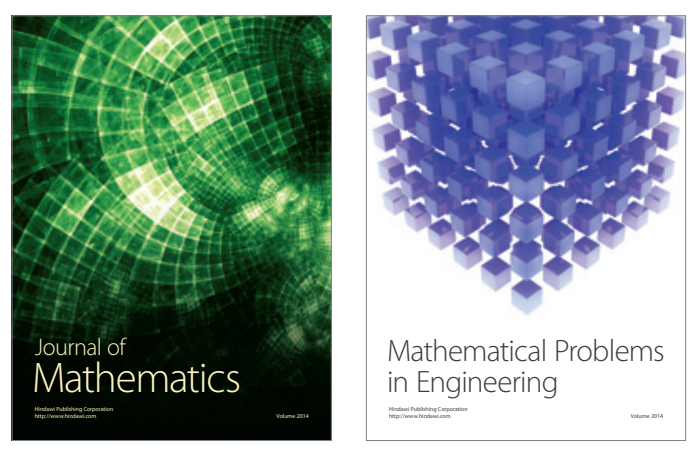

Mathematical Problems in Engineering
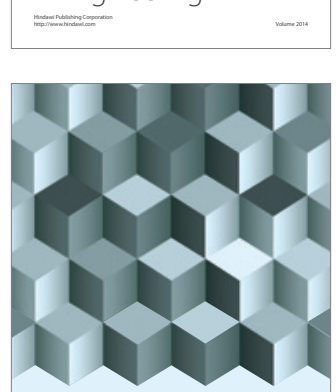

Journal of

Function Spaces
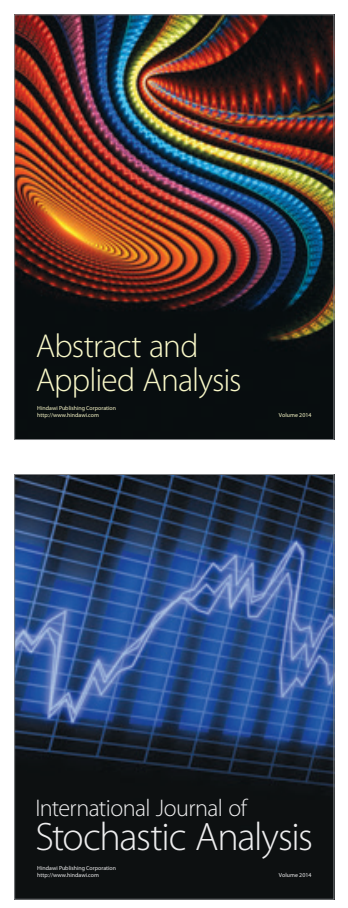

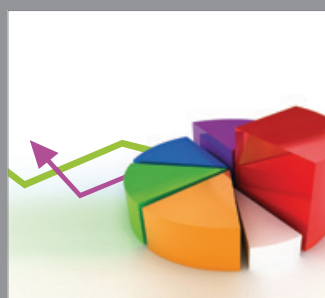

ournal of

Probability and Statistics

Promensencen
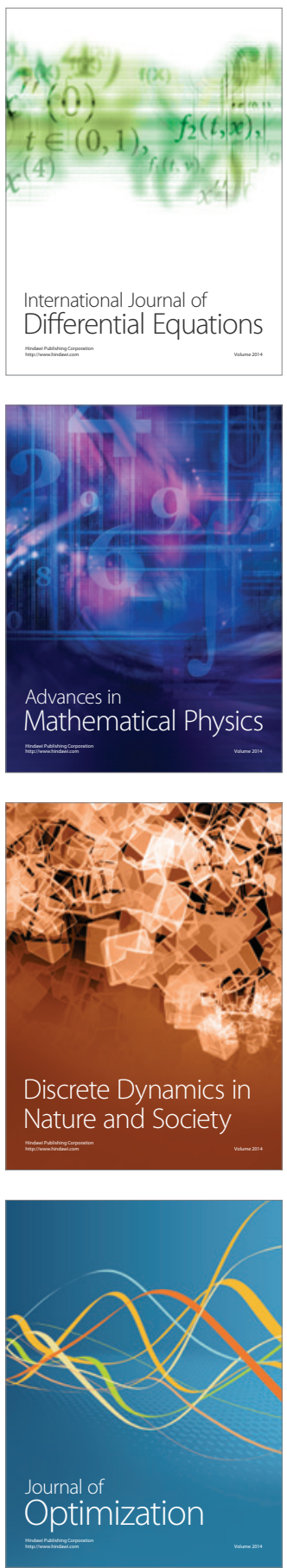